\title{
COMPARISON OF BIT ERROR RATE PERFormanCE OF VARIOUS Digital MODULATION SCHEMES OVER AWGN AND RAYLEIGH FADING CHANNELS USING SIMULINK
}

\author{
Md. Firoz Ahmed ${ }^{1}$, Md. Faysal Ahmed ${ }^{1}$ and Abu Zafor Md. Touhidul Islam² \\ ${ }^{1}$ Department of Information and Communication Engineering, \\ Rajshahi University, Rajshahi-6205 Bangladesh \\ ${ }^{2}$ Department of Electrical and Electronic Engineering, Rajshahi University, \\ Rajshahi-6205 Bangladesh
}

\begin{abstract}
Digital modulation increases information capacity, data security, and system availability while maintaining high communication quality. As a result, digital modulation techniques are in higher demand than analog modulation techniques due to their ability to transmit larger amounts of data. Amplitude Shift Keying (ASK), Frequency Shift Keying (FSK), Phase Shift Keying (PSK), Differential Phase Shift Keying (DPSK), and Quadrature Amplitude Modulation (QAM) are critical components of current communications systems development, particularly for broadband wireless communications. In this paper, the comparison of bit error rate performance of different modulation schemes (BPSK, QPSK, and16-QAM) and various equalization techniques such as constant modulus algorithm (CMA) and maximum likelihood sequence estimate (MLSE) for the AWGN and Rayleigh fading channels is analyzed using Simulink. BPSK outperforms QPSK and 16-QAM when compared to the other two digital modulation schemes. Among the three digital modulation schemes, BPSK is showing better performance as compared to QPSK and 16$Q A M$.
\end{abstract}

\section{KEYWORDS}

AWGN Channel, Rayleigh Fading Channel, CMA, MLSE

\section{INTRODUCTION}

The performance of transmitting and receiving systems is critical in recent times for rapidly growing wireless technologies. Digital modulation schemes help to advance mobile communications by increasing wireless network capacity, speed, and quality [1]. The strategies of digital modulation increase information-carrying capacity, communication quality, data security, and RF spectrum sharing, allowing for more services. The choice of digital modulation scheme will significantly affect the characteristics, performance, and resulting physical realization of a communication system. There is no universal 'best' scheme, but some will be a better fit than others depending on the physical characteristics of the channel, required levels of performance, and target hardware trade-offs. The required data rate, acceptable latency, available bandwidth, anticipated link budget, and target hardware cost, size, and current consumption must all be taken into account [2].

The next-generation wireless communication systems require higher data transmission rates in order to meet the higher demand for quality services [3]. A major transition from analog to digital 
communications has occurred in the last few decades, and it can be seen in all fields of communication because a digital communication system is more reliable than an analog system. Digital modulation methods are preferred over analog modulation schemes because they provide greater immunity to noise at the expense of higher bandwidth requirements, whereas the requirement of video, audio, and data over a computer network or a mobile telephony network, referred to as third-generation mobile communication, poses a serious bandwidth problem, as a result, existing modulation schemes must be modified for the purpose so that they can handle both noise and bandwidth efficiency [4].

In comparison to analog transmission, digital transmission provides greater reliability in a noisy environment. However, sometimes the digital information, i.e. the transmitted pulses, is smeared out to the point where pulses corresponding to different symbols cannot be distinguished, a phenomenon known as inter-symbol interference (ISI) [5]. The bit error rate (BER) versus signalto-noise ratio (SNR) curve can be used to assess the channel's performance. In digital transmission, BER is defined as the percentage of bits with errors divided by the total number of bits transmitted, received, or processed in a given time period. Typically, the rate is expressed as 10 to the negative power. Unwanted energy is represented by noise. When the signal is weak, noise can interfere with it at any point in the communication system. The equalizer provides the inverse of the channel to the received signal, resulting in flat frequency response and linear phase. The static equalizer's noise performance is subpar. Most of the time, the transfer functions of the transmission system are unknown. In addition, the impulse response of the channel may vary over time. As a result, designing equalizers becomes difficult [6]. Because the channel has amplitude and phase dispersion, the transmitted signals interfere with one another. As a result, equalizers are created to address this issue. The equalizer is designed to work in such a way that the Bit Error Rate (BER) is low and the Signal-to-Noise Ratio (SNR) is high [7].

In [8], the performance of digital modulation schemes-BPSK, QPSK, and QAM-with MATLAB is investigated. They demonstrated that BPSK outperforms QPSK and QAM in both cases, using AWGN and Rayleigh fading channels. In [9], the bit error rate performance characteristics of the receiver are evaluated using a MATLAB Simulink model for BPSK, QPSK, BFSK, DBPSK, MPSK, M-FSK, and QAM modulation techniques. According to the study's findings, BPSK is the most effective modulation scheme in a practical communication system. The Simulink model is used to study and analyze the performance of stego image transmission in [10]. According to the result of the study, the PSNR graphs show that the best modulation scheme having higher error tolerance than others. A comparative analysis of various equalization techniques (LMS, RLS, and CMA) in the OFDM System using different Digital Modulations are introduced in [11]. According to the Simulink results, the CMA equalizer in the OFDM-based BPSK system produces the lowest BER value when compared to the LMS and RLS equalizers. In [12], the performance comparison of non-linear and adaptive equalization algorithms for wireless digital communication is presented. Adaptive algorithms like LMS and RLS are more preferable where channel characteristics are unknown a priori and, in many cases, the channel response is timevariant, according to studies.

The present study investigates the comparison of bit error rate performance of different digital modulation techniques are used over the AWGN channel and on Rayleigh fading with the implementation of CMA and MLSE equalizer schemes for 10000 bits transmission. 


\section{Equalizers}

\subsection{Constant Modulus Algorithm (CMA)}

The constant modulus algorithm (CMA) is a shortened form for the algorithm of constant modulus. To equalize a linearly modulated baseband signal passing through a dispersive channel, the CMA Equalizer block employs a linear equalizer and the constant modulus algorithm (CMA). The CMA is used by the block to update the weights once per symbol during simulation [13]. If the parameter Number of samples per symbol is set to 1, the block will implement a symbolspaced equalizer; otherwise, the block will implement a fractionally spaced equalizer [13].

\subsection{Maximum Likelihood Estimation}

Equalizers based on Maximum-Likelihood Sequence Estimation (MLSE) provide optimal equalization of time variations in propagation channel characteristics. MLSE equalizers, on the other hand, are sometimes less appealing because their computational complexity is higher than that of adaptive equalizers. The viterbi algorithm is used by the MLSE equalizer block to equalize a linearly modulated signal passing through a dispersive channel. These characteristics produce the maximum likelihood sequence estimate of the signal using a channel estimate modeled as a finite input response (FIR) filter. An MLSE equalizer provides the best theoretical performance but is computationally demanding. [14].

\section{Channels}

The most important aspect of any communication system is the channel. The performance of a communication channel is affected by noise. There are several types of communication channels.

\subsection{AWGN (Additive White Gaussian Noise) Channel}

Additive White Gaussian Noise is a fundamental noise model used in information theory to simulate the effect of many random processes found in nature. Modifiers are words that describe specific qualities.

The AWGN channel is a common channel model in which the only impediment to communication is a linear addition of wideband or white noise with constant spectral density and Gaussian amplitude distribution. Fading, frequency selection, interference, and dispersion are not taken into account in the model. Before considering these phenomena, it generates simple and tractable mathematical models that are useful for gaining insight into the underlying behavior of the system. It is an appropriate model for many satellite and deep space communications links. It's the most fundamental communication system model [15].

\subsection{Fading Channel}

The term "fading" refers to the rapid fluctuations in amplitude, phase, and multipath delays of a radio signal over a short period of time or travel distance, allowing large-scale path loss effects to be ignored. There are various types of fading channels depending on the circumstances; however, we will focus on Rayleigh fading channels, in which the impulse response may follow distributions of Rayleigh distributions (in which there is no Line of Sight (LOS) ray between transmitter and receiver [15]. 


\section{Proposed Model}

The proposed model of the communication system using various equalizers such as constant modulus algorithm (CMA) and maximum likelihood sequence estimation (MLSE) for different digital modulation schemes (BPSK, QPSK, and16-QAM) shown below in Fig.1.

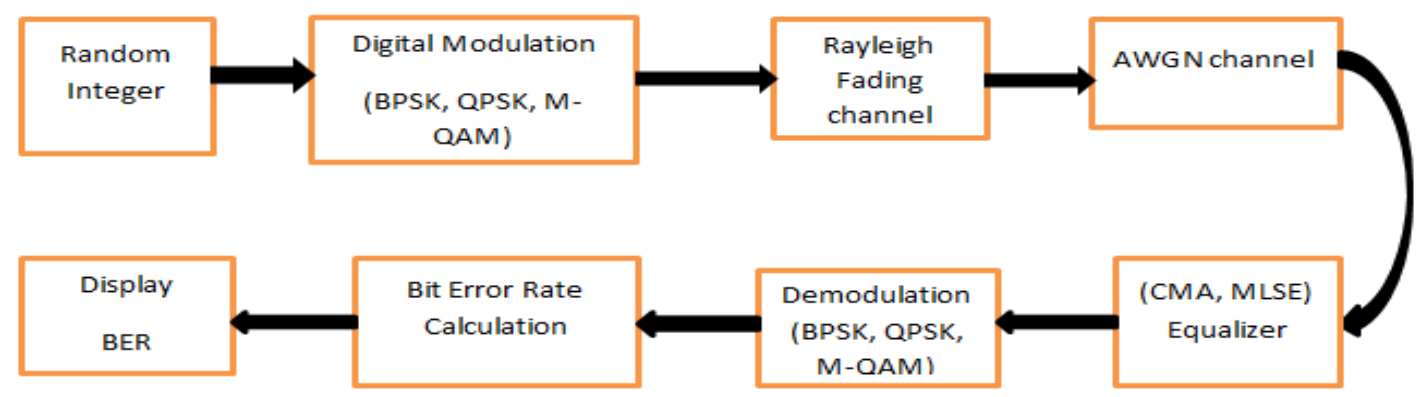

Figure 1. Proposed model of the communication system using various equalizers (CMA, MLSE) for different digital modulation schemes

The proposed model is made up of a transmitter, a channel, and a receiver. The data in this proposed work is generated using a random integer. Following that, the output is modulated using the digital modulation technique (BPSK, QPSK and 16-QAM). Following modulation, the output is routed through a multipath Rayleigh channel and an AWGN channel. The channel output is independently routed through the CMA and MLSE equalizers. After that, the equalized output is demodulated. The demodulated output is fed into an error rate calculator to determine the BER value. For CMA and MLSE equalization, the BER value is computed. A BER comparison for various digital modulation schemes is performed.

\section{Simulink ReSUlts AND ANALYSIS}

Matlab based Simulink is used to simulate the proposed communication system model, which employs CMA and MLSE equalization techniques. The simulation parameters are as follows:

Modulation - BPSK, QPSK and 16-QAM

Equalizer - CMA and MLSE

Fading channel - Multipath Rayleigh, AWGN

The BER results using the CMA equalizer and MLSE for the BPSK, QPSK, and 16-QAM modulation is shown in Fig. 2 to 7. 
International Journal of Ambient Systems and Applications (IJASA) Vol.9, No.1/2, June 2021

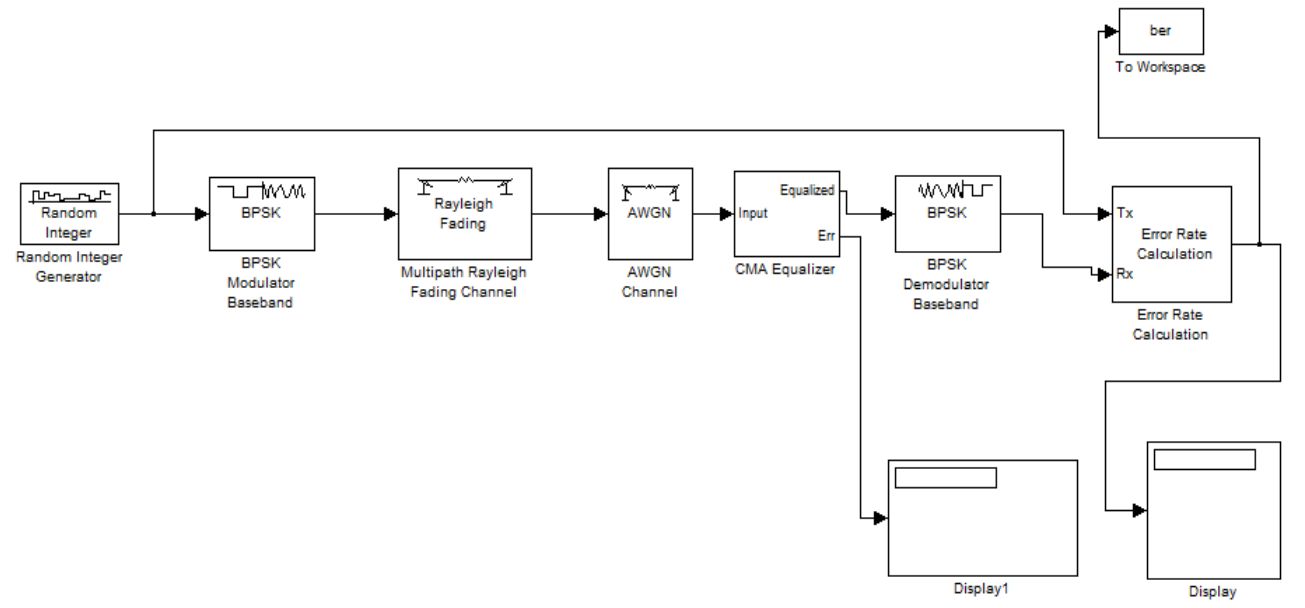

Figure 2. BER Analysis of CMA Equalizer for BPSK modulation

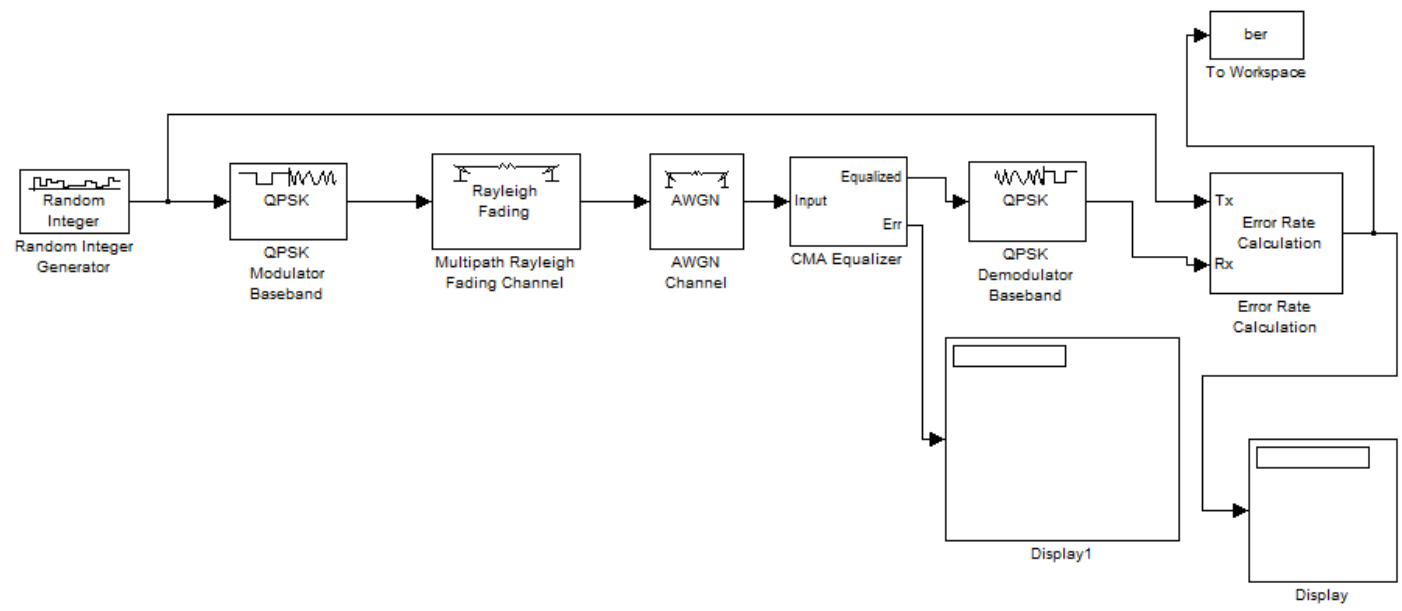

Figure 3. BER Analysis of CMA Equalizer for QPSK modulation

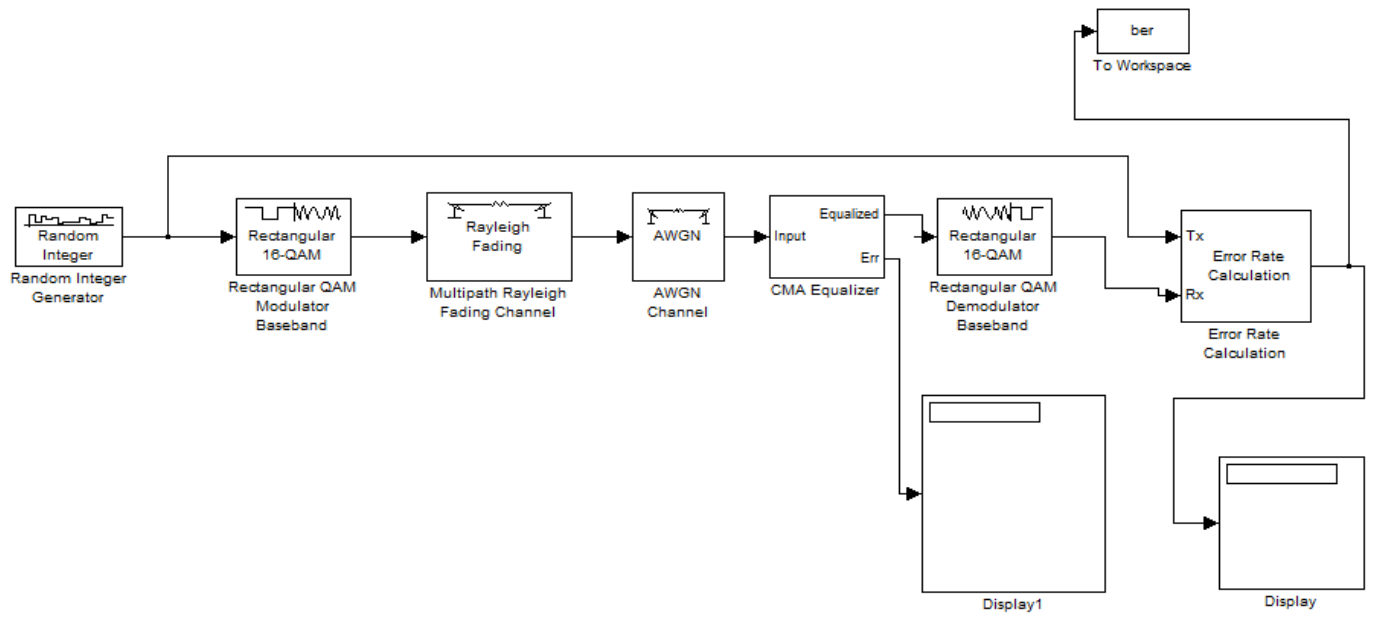

Figure 4. BER Analysis of CMA Equalizer for 16-QAM modulation 
International Journal of Ambient Systems and Applications (IJASA) Vol.9, No.1/2, June 2021

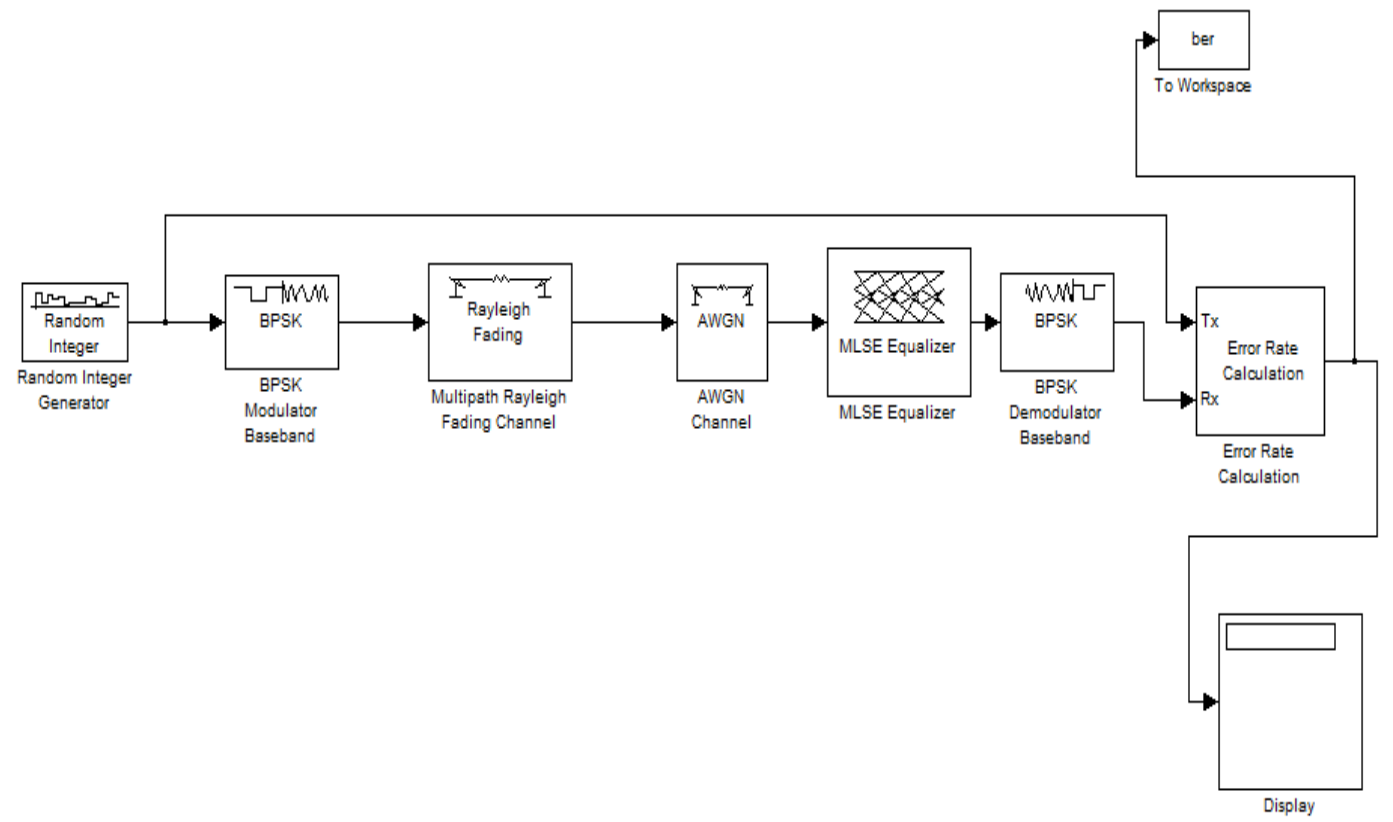

Figure 5. BER Analysis of MLSE Equalizer for BPSK modulation

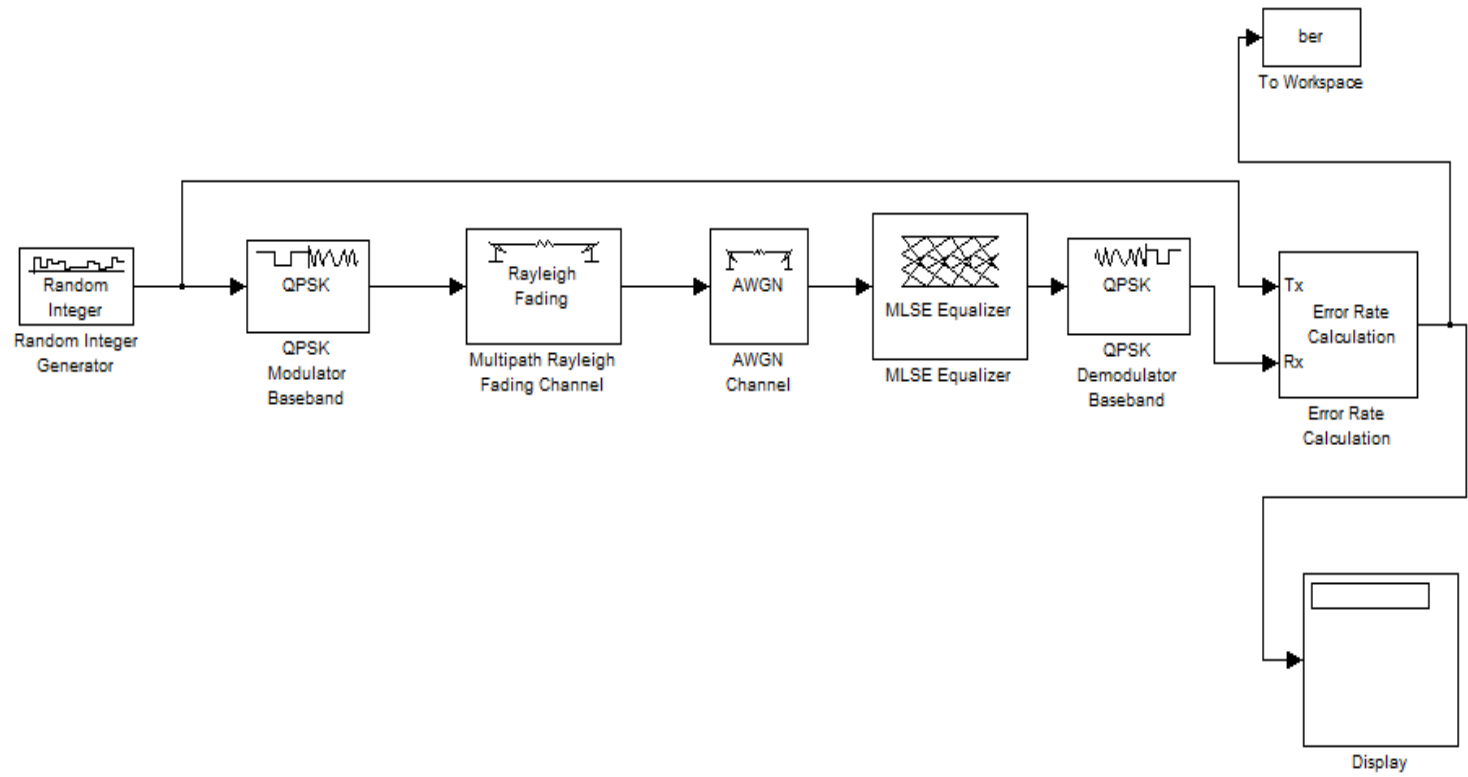

Figure 6. BER Analysis of MLSE Equalizer for QPSK modulation 


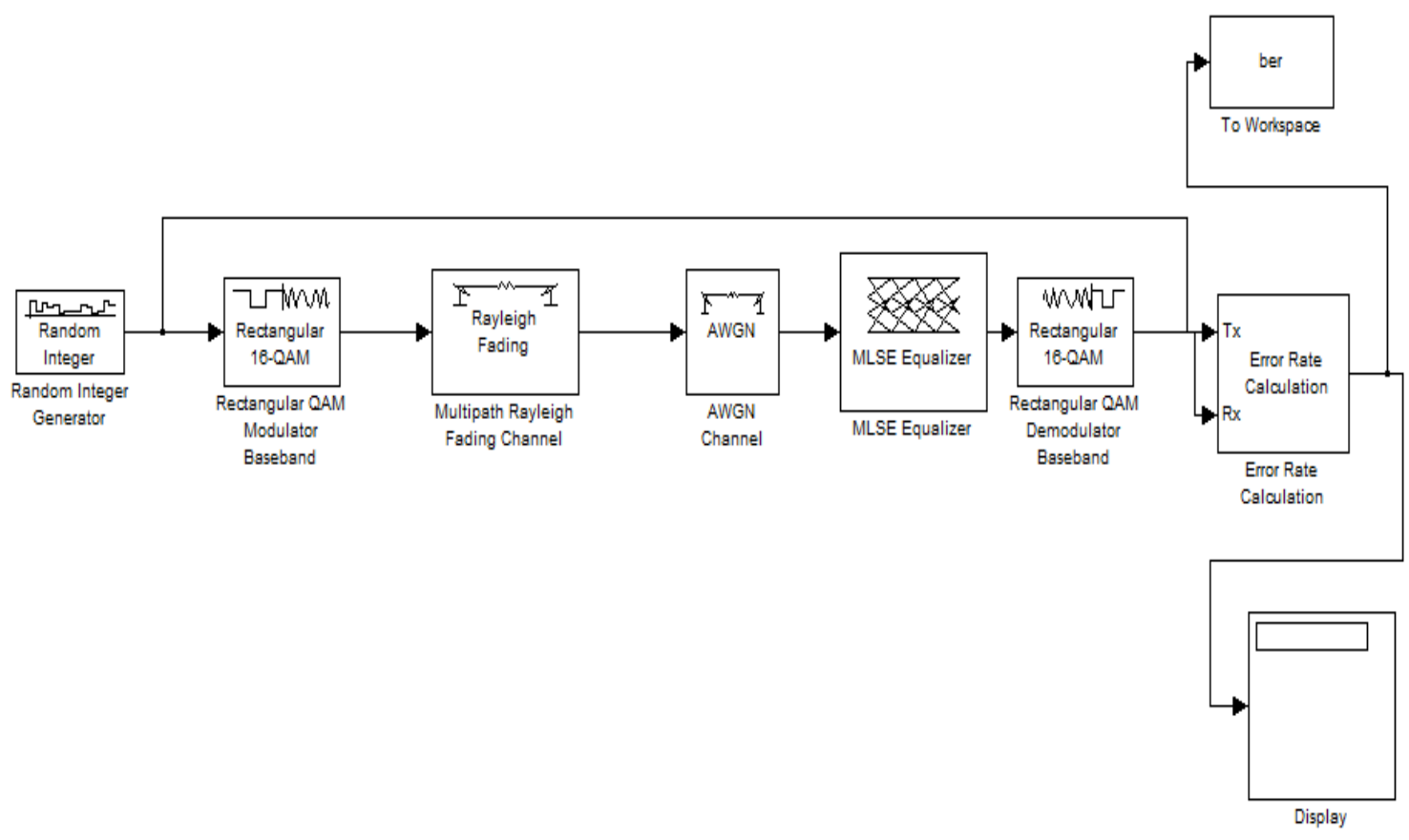

Figure 7. BER Analysis of MLSE Equalizer for 16-QAM modulation

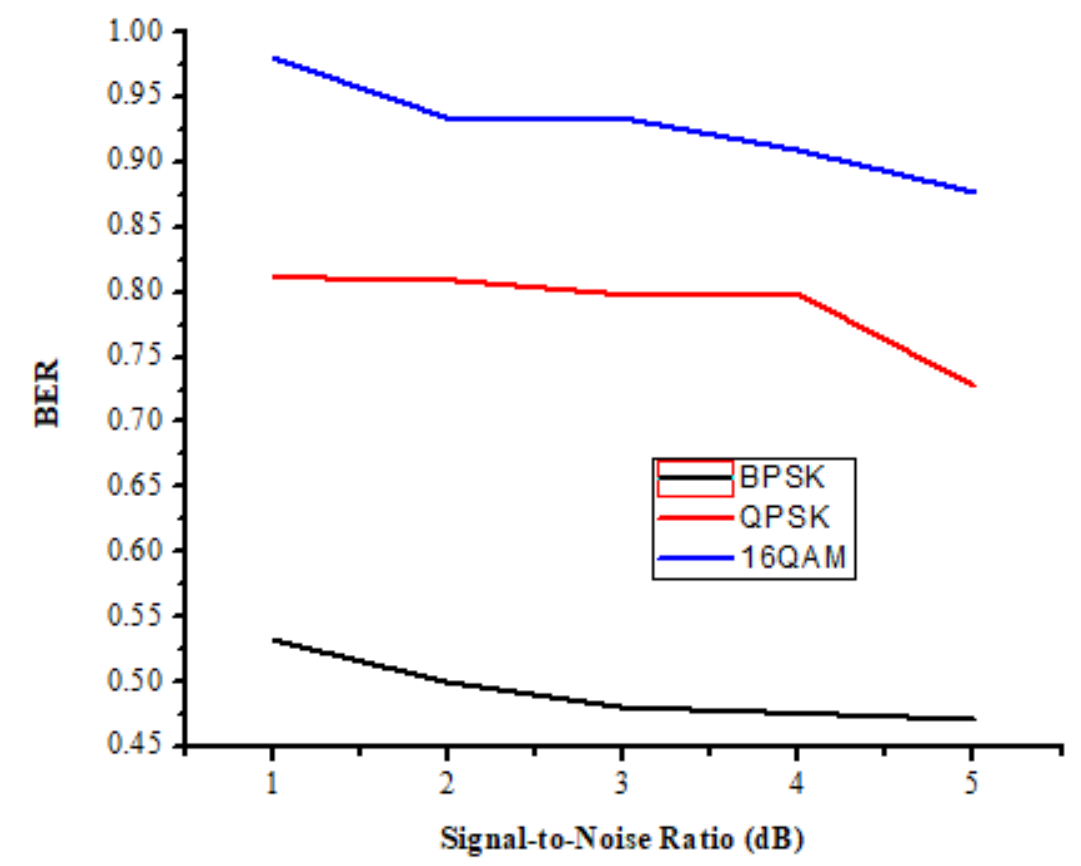

Figure 8. BER performance comparison of BPSK, QPSK and 16-QAM digital modulation schemes using CMA equalizer 


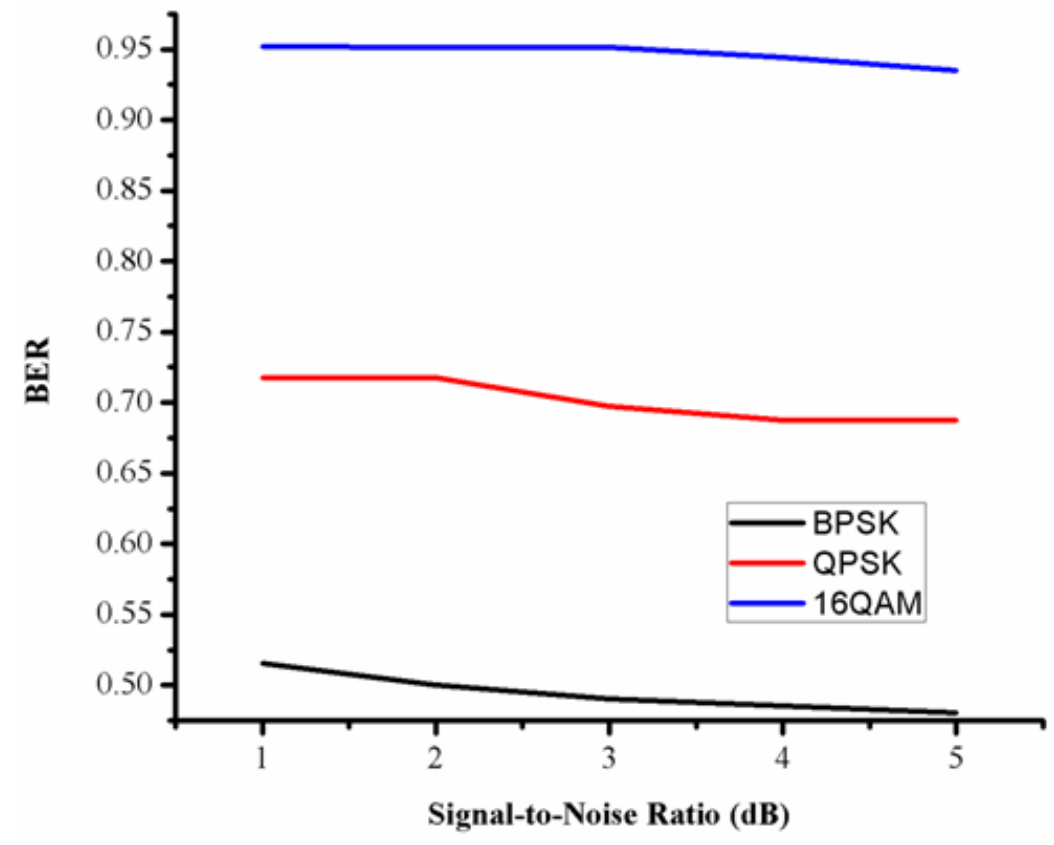

Figure 9. BER performance comparison of BPSK, QPSK and 16-QAM digital modulation schemes using MLSE equalizer

Fig. 8 represents a system performance comparison using a constant modulus algorithm (CMA)based equalizer scheme with various digital modulation methods (BPSK, QPSK, and 16-QAM). As shown in Table 1, the BER values for BPSK, QPSK, and 16-QAM digital modulation schemes are 0.4714, 0.7279, and 0.8772, respectively, in the general expected SNR value of 5 $\mathrm{dB}$, implying that the system performance achieves a gain of $10.25 \mathrm{~dB}, 8.37 \mathrm{~dB}$, and $7.56 \mathrm{~dB}$ using BPSK, QPSK, and 16-QAM strategies, respectively, with the implementation of CMA equalizer technique. When SNR is equal to 1 decibel, 16-QAM has a higher BER value than other modulation schemes. Table 1 gives the BER values of the system using the CMA equalizer method, as well as various digital modulation schemes (BPSK, QPSK, and 16-QAM) at various SNR levels $(1 \mathrm{~dB}-5 \mathrm{~dB})$. In terms of BER performance, the table clearly shows that for different SNR values $(\mathrm{SNR}=1 \mathrm{~dB}-5 \mathrm{~dB})$, BPSK outperforms QPSK and 16-QAM. The BER performance of the system is compared in Fig. 9 using BPSK, QPSK, and 16-QAM digital modulation schemes with an MLSE-based equalizer scheme. The bit error rate (BER) is much lower than the signal-to-noise ratio (SNR). The performance of BPSK is clearly superior to that of the others, as evidenced by this figure. QPSK, on the other hand, has a nearly smaller result, whereas 16-QAM has a higher bit error rate (BER). When $\mathrm{SNR}=1 \mathrm{~dB}, 16-\mathrm{QAM}$ has a higher BER value than other modulation techniques. Table 1 show the BER values of the system with the MLSE equalizer scheme and various digital modulation schemes (BPSK, QPSK, and 16-QAM) at different SNR $(1 \mathrm{~dB}-5 \mathrm{~dB})$. The table clearly shows that for different SNR values $(\mathrm{SNR}=1 \mathrm{~dB}-5 \mathrm{~dB})$, BPSK outperforms QPSK and 16-QAM in terms of BER performance.

According to the results of the preceding analysis, the system performs the worst in the MLSEbased equalizer scheme when compared to the CMA equalizer technique in 10000 bits transmission. 
International Journal of Ambient Systems and Applications (IJASA) Vol.9, No.1/2, June 2021

Table 1. BER results of 10000 bits transmission of CMA and MLSE equalizer for different digital modulation schemes.

\begin{tabular}{|c|c|c|c|c|}
\hline \multirow{7}{*}{ CMA } & \multirow[b]{2}{*}{$\begin{array}{l}\text { Signal-to } \\
\text { Noise } \\
\text { Ratio } \\
\text { dB }\end{array}$} & \multicolumn{3}{|c|}{ Bit Error Rate (BER) } \\
\hline & & BPSK & QPSK & 16-QAM \\
\hline & 1 & 0.5323 & 0.8115 & 0.9804 \\
\hline & 2 & 0.5000 & 0.8095 & 0.9340 \\
\hline & 3 & 0.4806 & 0.7984 & 0.9340 \\
\hline & 4 & 0.4762 & 0.7984 & 0.9091 \\
\hline & 5 & 0.4714 & 0.7279 & 0.8772 \\
\hline \multirow{5}{*}{ MLSE } & 1 & 0.5156 & 0.7174 & 0.9519 \\
\hline & 2 & 0.5000 & 0.7174 & 0.9515 \\
\hline & 3 & 0.4902 & 0.6972 & 0.9512 \\
\hline & 4 & 0.4854 & 0.6875 & 0.9444 \\
\hline & 5 & 0.4808 & 0.6875 & 0.9352 \\
\hline
\end{tabular}

\section{CONClusion}

In this paper, the comparison of bit error rate performance of different modulation schemes (BPSK, QPSK, and 16-QAM) and various equalization techniques (CMA and MLSE) for the AWGN and Rayleigh fading channels has been successfully analyzed using Simulink. A variety of modulation schemes, including BPSK, QPSK, and 16-QAM, were considered for simulation and their BER was evaluated using the MATLAB based Simulink simulation tool for Rayleigh fading and the Additive White Gaussian Noise Stream. According to the Simulink results in the preceding table, BPSK is the most efficient modulation scheme for CMA equalizer in a practical communication system, as compared to MLSE equalizer, depending on the bit error rate (BER).

\section{REFERENCES}

[1] M. Barnela, "Digital Modulation Schemes Employed in Wireless Communication: A Literature review," International Journal of Wired and Wireless Communications, Vol.2 (2) (April, 2014).

[2] Geoff Smithson, "Introduction to Digital Modulation Schemes", IEE Colloquium on The Design of Digital Cellular Handsets, London, UK, page(s): 2.1-2.9 (1998).

[3] J.T. Haitham, and M.F.M Salleh, "Multi-carrier Transmission Techniques for Wireless Communication Systems: A Survey, WSEAS Transactions on Communications," ISSN: 1109-2742, Vol.8 (5) (May 2009).

[4] R. Pandey, and K. Pandey, "An Introduction of Analog and Digital Modulation Techniques in Communication System," Journal of Innovative Trends in Science Pharmacy \& Technology, Vol. 1 (2014).

[5] A.C.Gurve, S.S. Wasnik, A.Yerlekar, and N. Chide, "Study of OFDM Variants and Implementation of OFDM Using fft/ifft," International Journal of Advanced Research in Computer and Communication Engineering, Vol. 3(7) (July 2014).

[6] B.W.Luo, X.Y. Zhang, and X.K. Ren, "On Applications of OFDM Technique," Video Engineering. Vol. 2, pp. 005, (2006). 
International Journal of Ambient Systems and Applications (IJASA) Vol.9, No.1/2, June 2021

[7] N.Marchetti, M.I.Rahman, S. Kumar, and R. Prasad, "OFDM: Principles and challenges. In New directions in wireless communications research," Springer, US. Pp.29 - 62, (2009).

[8] R. Prameela Devi, and H.Nishat,"Performance Evaluation of Digital Modulation Schemes BPSK, QPSK \& QAM, "International Journal of Engineering and Techniques, Vol.3 (2), (2017).

[9] M.G.Sadeque, "Bit Error Rate (BER) Comparison of AWGN Channels for Different Type's Digital Modulation Using MATLAB Simulink, "American Scientific Research Journal for Engineering, Technology, and Sciences (ASRJETS) Vol.13(1), pp 61-71,(2015).

[10] R.Tiwari1, and M.R.Mishra, "Comparative Analysis of Stego Image Transmission through OFDM Channel: A Simulink Model," International Journal of Science, Engineering and Technology Research (IJSETR), Vol.4 (5), (May 2015).

[11] P.Manhas, and M.K Soni, "Comparison of Various Channel Equalization Techniques in OFDM System using different Digital Modulations," Indonesian Journal of Electrical Engineering and Computer Science, Vol.3 (3), pp. 634 638, (September 2016).

[12] J.Bhalani, A.I.Trivedi, Y.P.Kosta, and V. T. Patel "Performance Comparison of Non-Linear and Adaptive Equalization Algorithms for Wireless Digital Communication," First Asian Himalayas International Conference on Internet, (2009).

[13] K. Elangovan, "Comparative study on the channel estimation for OFDM system using LMS, NLMS and RLS algorithms," In Pattern Recognition, Informatics and Medical Engineering (PRIME). International Conference on IEEE. pp. 359-363 (2012).

[14] MLSE Equalizers Retrieved from MATLAB \& Simulink.

[15] Er.M.Maheswari, S.Selvabharathi, and V.Subasri, "An End to End Simulation of Wireless Communication through AWGN and Fading Channel, "International Journal of Creative Research Thoughts (IJCRT), Vol.8 (4), (April 2020). 